\title{
Wanted: a Transdisciplinary Knowledge Domain for Urban Health
}

\author{
Roderick J. Lawrence (ID - Franz W. Gatzweiler
}

Published online: 10 July 2017

(C) The New York Academy of Medicine 2017

\begin{abstract}
The current disconnection between access to increasing amounts of data about urbanization, health, and other global changes and the conflicting meanings and values of that data has created uncertainty and reduced the ability of people to act upon available information which they do not necessarily understand. We see a disconnection between increasing data availability and data processing capability and capacity. In response to this disconnection, modeling has been attributed an important role in international and national research programs in order to predict the future based on past and recent trends. Predictive models are often data heavy and founded on assumptions which are difficult to verify, especially regarding urban health issues in
\end{abstract}

\section{R. J. Lawrence}

Geneva School of Social Sciences (G3S), University of Geneva, Geneva, Switzerland

e-mail: roderick.lawrence@unige.ch

\section{R. J. Lawrence}

School of Architecture and the Built Environment, Faculty of the Professions, University of Adelaide, Adelaide 5005, Australia

\section{R. J. Lawrence}

Institute for Environment and Development (LESTARI), Universiti Kebangsaan Malaysia (UKM), Bangi, Selangor, Malaysia

\section{F. W. Gatzweiler $(\bowtie)$}

International Council for Science (ICSU), Urban Health and Wellbeing: a Systems Approach International Programme Office, Institute of Urban Environment, Chinese Academy of Sciences,

Xiamen, China

e-mail: gatzweiler02@gmail.com specific contexts. Producing large volumes of data warrants debate about what data are prerequisites for better understanding human health in changing urban environments. Another concern is how data and information can be used to apply knowledge. Making sense of empirical knowledge requires a new transdisciplinary knowledge domain created by a commitment to convergence between researchers in multiple academic disciplines and other actors and institutions in cities. Disciplinary-based researchers are no longer the sole producers of empirical knowledge. Today, diverse kinds of knowledge are becoming an emergent product of multiple societal stakeholders acting collectively to address challenges that impact on their habitat, their livelihood, and their health. Insights from complexity science also require a fundamental rethinking of the role and responsibility of human agency while admitting rather than denying complexity and radical uncertainty.

\section{Commentary}

We have great difficulty in understanding the complex consequences of how humans transformed the Earth over several millennia in order to construct their habitat and sustain their livelihood. We also have difficulty in understanding how societies have positively and negatively influenced the habitat of all living organisms on Earth. The term global change is not informative about the diversity and multiple consequences of these impacts especially on human health in specific localities, including cities around the world. 
Scientific research has difficulty in identifying and measuring the total impacts and consequences of global change, notably climate change; loss of biodiversity; and urbanization on our human habitat, livelihoods, and health. For example, we are informed by the media that a $2^{\circ}$ increase in the average surface temperature of the Earth will reduce the number of days for downhill and cross-country skiing in many winter resorts or increase the number of days with high temperatures in many cities. However, we do not know whether our habitat, livelihood, and health are vulnerable, or whether the impacts of climate change are imminent, inevitable, or reversible. The behavioral responses of citizens confronted by complexity include seeking ways of trying to know how change will impact on their habitat and livelihood, or alternatively by living in denial: "This... has implications for our conceptions of individual caring and intelligence, for social cognition, for the role of culture in reproducing social relations, and for the centrality of emotions in culture, cognition, and political action" [1].

The key question that scientists, policy makers, and professionals therefore need to address is What kinds of information are necessary in order for citizens to better understand how a plausible temperature increase of $2^{\circ}$ could impact on their habitat, health, and livelihood, and whether that is actually the relevant question, in and for specific cities. At the outset, it is important to note that such questions cannot be answered simply by a quest for more data, geo-statistics and predictive models.

Global environmental, economic, and social changes are the result of multiple and reciprocal relations between highly complex systems in the biosphere. The multiple components of the Earth's diverse ecosystems include human societies, their habitat, culture, social organization, and economy. The coexistence and mutual interaction between the human and non-human constituents of ecosystems across several eco-geo-political levels has been the subject of human ecology for decades [2]. Of particular relevance are the dynamic, mutual interactions between the abiotic, biotic, and anthropologic components of ecosystems, as well as the distinction between those components that constitute structure and those that change structure [3]. Human ecology admits the complexity, diversity, systemic, and unpredictable nature of ecosystems including the dynamic interrelations between their human and nonhuman components over time.
Therefore, it should not be assumed that ecosystems only conform to the rule-based conception of Newtonian science. This common interpretation of science is based on observation, experimentation, falsification, replication, and verification. These procedures can lead to the identification of laws and control parameters. These are formulated in order to assert certainty for decision making and support the limited abilities of the human mind to grasp complexity. They also follow the human desire to expand current knowledge about real world challenges in order to respond to them [4]. This analytical approach has often been applied in research that represents or simulates global change, especially climate change, by simulation models. Much research has concentrated on identifying and measuring human impacts on the biosphere and the Earth, especially its specific components (e.g., the atmosphere, the oceans, or terrestrial ecosystems). Predictive modeling has been attributed an important role in international and national research programs in order to forecast the future based on past and recent trends. Such predictive models are often founded on at least three assumptions that warrant critical rethinking:

First, many modelers assume that changes in both human and non-human ecosystems on Earth can be studied and understood in finite predictable ways by systematic observation, measurement, and data analysis over time. This assumption overlooks complexity and the unpredictable nature of change in dynamic systems on Earth and in the biosphere [5].

Second, modelers often assume that the interactions between the parts of systems on Earth obey the same functions and processes around the world, to the extent that there is no intrinsic variability in the functions of natural and human-made ecosystems. This assumption ignores biological and cultural diversity and the variable capacity of humans and other biological species to adapt to change [6].

Third, it is assumed that complex phenomena on Earth, such as atmosphere-ocean coupling for the sequestration of carbon, can be forecast on the basis of how these complex systems have functioned and evolved over time, whereas the probability of change in individual and collective human behavior is often discounted [7].

These three basic assumptions raise fundamental issues. Those researchers who wish to understand the complexity of the Earth's multiple systems and how they change over time are seeking a degree of certainty 
to support decision making by actors and institutions in the public and private sectors. However, this reductionist approach cannot account for the unpredictability of change in the Earth's multiple systems. Hence, the future cannot be precisely prescribed because there will always be a degree of uncertainty. Therefore, decisions are often made on false assumptions about the quantification and predictability of change. One result is that unforeseen and unintended consequences of decisions occur, as shown by public policies for land use planning and urban development in many countries $[8,9]$. Admittedly, this has led many modelers to develop alternative scenarios of plausible futures. However, the basic problem is compounded by the fact that these scenarios are formulated using the same assumptions which frequently remain implicit and unstated.

Today, there are numerous ways of recording global change including climate change, biodiversity loss, and migration flows. Geo-spatial data sets at global, regional, national, and sub-national levels are now accessible to users around the world. The biophysical and mathematical sciences are committed to improving our understanding of the geophysical and biochemical dimensions of climate change. However, there can be significant cultural, institutional, and political barriers to collaboration and coordination across these interrelated geo-political levels, as the challenge of climate change has clearly illustrated. These challenges are barriers to our scientific and societal data processing capabilities.

We must also understand the individual, group, and societal attitudes, perceptions, motivations, reasoning, and values concerning climate change at each of these levels. Only then can we consider which behavioral, financial, political, and technological tools can be applied in specific situations to deal with urban heath challenges. These fundamental human dimensions have not adequately been addressed in recent research on global change and sustainability. This is regrettable because these dimensions are the foundations of human agency that can explain collective action to respond to the impacts of global change in specific localities [10].

The purpose of producing large volumes of data and other kinds of quantitative information warrants debate in our so-called information society. It is pertinent to ask what data and information are prerequisites for better understanding global change, especially climate change, loss of biodiversity, and urbanization and how impacts on habitats, health, and livelihoods in specific localities can or should be estimated quantitatively. We still lack data and information about our dominant habitat, the city, and its urban ecosystems. We need stronger commitment for the systematic monitoring of urban ecosystems. We should also communicate this data and information about cities and population health to all concerned in ways so that people understand the key issues at stake and the interrelations between them. What data and information are necessary for elected officials, public administrators, private entrepreneurs, and community associations to make informed decisions across conventional sectors? In short, making sense of the data we have and continue to collect requires better scientific and societal data processing capabilities.

Making sense of empirical knowledge requires a new transdisciplinary knowledge domain created by a commitment to convergence between multiple academic disciplines in the natural and social sciences and the humanities and between practitioners and other actors and institutions in cities [11]. In an age of big data, disciplinarybased researchers are no longer the sole producers of empirical knowledge. Today, diverse kinds of knowledge are becoming an emergent product of multiple societal stakeholders acting collectively to address challenges that may impact on their habitat, livelihood, and their health. The challenge is to coordinate the expert knowledge for producing collective intelligence.

Climate change, loss of biodiversity, and urbanization are not just complex ecological phenomena; indeed, they are societal ones that concern sustaining human life and health in diverse climatic, cultural, geographical, and political contexts. Tackling the negative impacts of urbanization (including loss of biodiversity, access to water, and food security) will require a fundamental rethinking of the role and responsibility of human agency.

Human agency is the ability of individuals and groups to act knowingly on the basis of what they value in order to maintain or improve their well-being [12]. When actors and institutions are involved in concerted actions to tackle real-world challenges, the power of some participants to influence or control the actions of others will need to be addressed. The lack of attention to the hidden agendas of participants in collaborative projects about sustainability needs correcting [13].

Transdisciplinarity has been endorsed by Future Earth, a large research platform, facilitated by the International Council for Science (ICSU) that is concerned by the impacts of global change and transformations to a more sustainable world. This international consortium has 
recently defined the content of transdisciplinary contributions as "the active involvement of researchers and stakeholders during the entire research process." This is what is also known as "co-designed and co-produced research" (http://www.futureearth.org/impact). This kind of collaboration means that researchers propose and coordinate methodologies, whereas the definition and analysis of the research questions and the dissemination of results are done jointly with other non-academic representatives of society.

Transdisciplinary projects on climate change, loss of biodiversity, and urbanization (and other global changes) extend beyond common research questions about the occurrence and magnitude of change represented by different kinds of models [14]. They address other equally important questions, such as how change is experienced by different groups or populations, why some countries have failed to acknowledge urbanization in national policy agendas, and how adaptation and resilience could become more effective. Human actions can result from admitting the radical uncertainty of complex urban ecosystems, the unpredictable changes that emerge from these systems, and the fact that humans are integral components of these ecosystems. Portugali presents two approaches that can implement agency in the context of complex challenges [15]. First, understanding complexity, then identifying control parameters, before modeling and simulating urban change. Second, participative urban planning can encourage the collaboration of many actors and institutions. Both these approaches have been referred to and elaborated in a "systems approach to urban health and wellbeing" [16].

Participative urban planning can address uncertainty and the limits to knowledge. It is a human-centered approach founded on the principle that humans are part of the system they want to understand. In doing so, humans define their own behavior by making new rules with regard to what they value doing and being in every-day life. Humans become rule-makers (creators) not only ruletakers by responding to their understanding of complex issues. Our understanding of complex systems remains incomplete if we do not account for the diverse ways that humans behave. Human agency makes the system complex, dynamic, and uncertain. Understanding the contribution of human beings in any ecosystem is a cognitive process. Creating the system by making rules is what is referred to as self-organization or self-creation (autopoiesis). Human agency can therefore be understood as a consequence of self-organization in complex systems.
The current disconnection between the availability of increasing amounts of data about urbanization (and other global changes) and the diverse sometimes conflicting meanings and values of that data has created uncertainty. This ongoing trend has reduced the ability of people to act upon the information they observe but do not necessarily understand. While data availability increases, capabilities of processing this data and assigning values and meaning to information do not increase accordingly. For example, the heat island effect produced by higher ambient temperatures in cities can have positive health impacts in colder seasons but negative health impacts during extended periods of high ambient temperatures. This is still not understood comprehensively by many citizens. It is legitimate to ask how scientific knowledge and modeling expertise can be more effectively used to reduce the gap between knowledge domains, policy domains, and everyday life.

A transdisciplinary knowledge domain is urgently required to address the complexity of urban health challenges and formulate effective responses to them. This kind of knowledge domain explicitly addresses the interface between scientific knowledge, public policies, and human behavior. The concept of a transdisciplinary knowledge domain, shown in Fig. 1, can help to redefine the interface between scientific knowledge, professional expertise, and citizens in an era when access to data and information has rapidly evolved. Innovative communication media for data and information provide new opportunities for collaboration between actors and institutions in all areas of contemporary society. For example, Fig. 1 shows that stronger collective action and intelligence [17] can bring applicable knowledge and information closer to each other. Figure 1 also shows that stronger communication and interaction can create shared understanding and attribute collective meaning to data - thereby improving the ability of citizens to understand how global change could impact on their habitat, health, and livelihood.

Human societies have used their collective intelligence and resources to construct a world of artifacts including their urban habitat. We need to admit that human creativity has both positive and negative consequences for the world in which we live. Humans have the capacity to change their habitat, their livelihood, and their impacts on the Earth's multiple systems. It is fundamentally important to reconsider the human capacity to transform this world for the better of current and future generations [18]. 


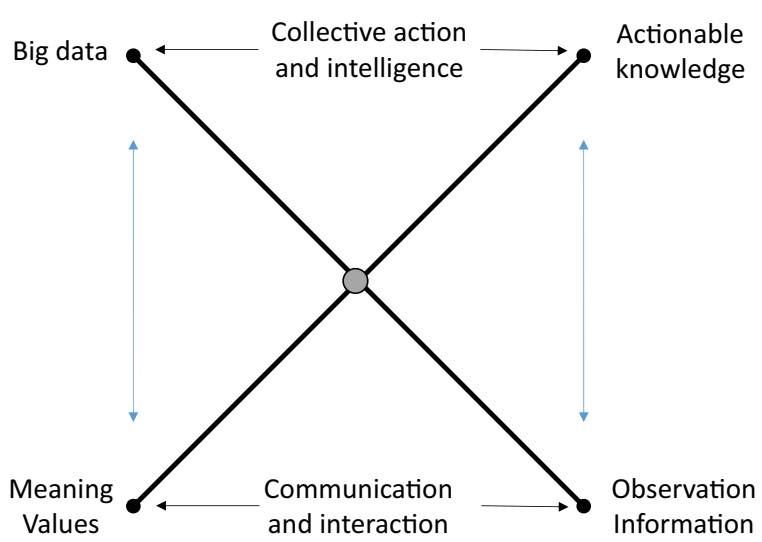

Fig. 1 Transdisciplinarity improves communication, collective action, and intelligence building for urban health and wellbeing

The fundamental shift to a transdisciplinary knowledge domain is founded on the principle that scientific knowledge (and representations of it by modelers) are only one component of a much larger societal knowledge domain that includes multiple types of quantitative and qualitative information, involving diverse concepts, meanings, methods, and values that can be shared and mutually understood by communication media and dia$\log$ platforms. This can be the framework for the coproduction of innovative concerted action by local communities in order to reduce the applicability gap between what is known, what is understood, and what is achieved in response to global challenges such urbanization [19]. Indeed, a plausible way forward to address the challenge of promoting the health of all urban populations.

\section{References}

1. Norgaard K. Living in denial. Climate change, emotions, and everyday life. Cambridge, MA: MIT Press; 2011. p. 207.

2. Lawrence R. Human Ecology. In: Tolba M, editor. Our fragile world: challenges and opportunities for sustainable development, volume 1. Oxford, UK: EOLSS Publishers; 2001. p. 675-93.

3. Dyball R, Newell B. Understanding human ecology: a systems approach to sustainability. Abington, UK and New York, NY: Routledge; 2015.
4. Brown V, Harris J, Russell J. Tackling wicked problems through transdisciplinary imagination. London, UK: Earthscan; 2010.

5. Pearson R, Dawson T. Predicting the impacts of climate change on the distribution of species: are bioclimate envelope models useful? Glob Ecol Biogeogr. 2003;12:361-71.

6. World Health Organization (2004). Using climate to predict infectious disease outbreaks: a review. Geneva, Switzerland: World Health Organization, document WHO/SDE/OEH/ 04.01 (English only).

7. Holling C. Understanding the complexity of economic, ecological and social systems. Ecosystems. 2001;4:390-405.

8. Rodwin L, Sanyal B. The profession of city planning: changes, images and challenges, 1950-2000. New Brunswick, NJ: Rutgers University Press; 2000.

9. Kresl P. Planning cities for the future: the successes and failures of urban economic strategies in Europe. Cheltenham, UK: Edward Elgar; 2007.

10. Brosch T. In: Sanders D, editor. Handbook of value perspectives from economics, neuroscience, philosophy, psychology and sociology. Oxford, UK: Oxford University Press; 2016.

11. National Academy of Sciences. Convergence: facilitating transdisciplinary integration of life sciences, physical sciences, engineering, and beyond. Washington DC: National Academy of Sciences; 2014.

12. Giddens A. The constitution of society: outline of a theory of structuration. Cambridge: Polity Press; 1986.

13. Rosendahl J, Zanella M, Rist S, Weigelt J. Scientists' situated knowledge: strong objectivity in transdisciplinarity. Futures. 2015;65:17-27.

14. Sheppard S. et al. Future visioning of local climate change: a framework for community engagement and planning with scenarios and visualization. Futures. 2011;43:400-12.

15. Portugali J. Self-organization and the city. Berlin, Germany: Springer; 2000.

16. Gatzweiler, F.W., Ayad, H.M., Boufford, J.I., Capon, A., Diez Roux, A.V., Donnelly, Ch., Hanaki, K., Jayasinghe, S., Nath, I., Parnell, S., Rietveld, L., Ritchie, P., Salem, G., Speizer, I., Zhang, Y., Zhu, Y-G. (2016). Advancing urban health and wellbeing in the changing urban environment. Implementing a Systems Approach. Zhejiang University Press and Springer.

17. Atlee, T. (2006). Co-intelligence, collective intelligence, and conscious evolution. In: Tovay, M. (ed.) Collective Intelligence: Creating a Prosperous World at Peace. Earth Intelligence Network, pp.1-4.

18. Whitmee, S., Haines, A., Beyrer, C., et al. (2015). Safeguarding human health in the anthropocene epoch: report of The Rockefeller Foundation-Lancet Commission on planetary health. Lancet published online July 16 . doi:10.1016/S0140-6736(15)60901-1

19. Brown V, Lambert J. Collective learning for transformational change. New York, NY: Earthscan; 2013. 